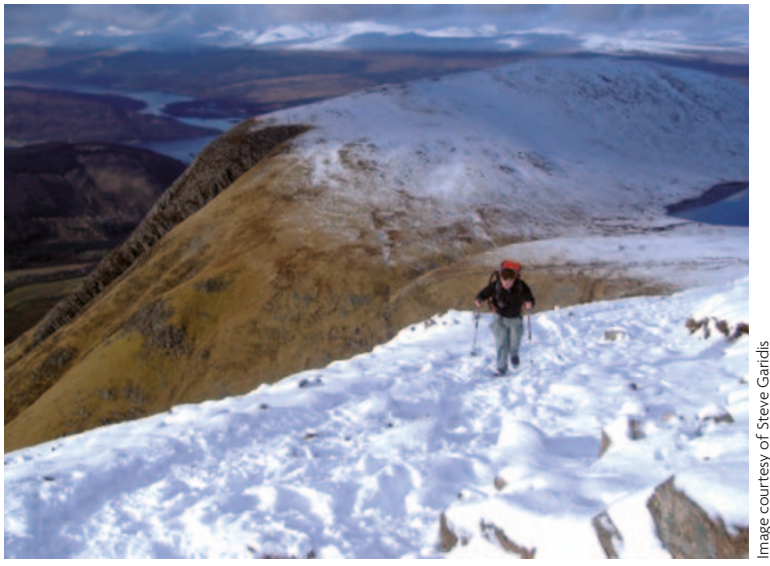

One of the central aims in neuroscience is to understand the molecular mechanisms of learning. Minichiello and colleagues have now addressed this question with a novel combination of in vivo methods and were able to show that signalling through the TrkB receptor and its PLC $\gamma$ binding site is important for associative learning and parallel longterm potentiation (LTP).

The TrkB receptor is well known for its importance in synaptic plasticity and LTP in the hippocampus. It mediates downstream signalling through the Ras/MAPK pathway through binding of Shc, or the calcium/calmodulin pathway through binding of PLC $\gamma$. Previous in vitro

\title{
$\Rightarrow$ LEARNING AND MEMORY
}

\section{Learning through Trk-ing}

studies have shown that the PLC $\gamma$ but not the Shc - docking site of TrkB is crucial for hippocampal LTP. To investigate the question of whether learning is based on the same molecular mechanisms as LTP, the authors used an associative learning paradigm combined with direct in vivo recordings from the hippocampus of transgenic mice.

First, they tested transgenic mice carrying point mutations of the TrkB receptor in the Shc $\left(\operatorname{trk}^{\mathrm{SHC} /+}\right)$ or the PLC $\gamma\left(\operatorname{trk}^{\mathrm{PLC} /+}\right)$ docking site for their ability to learn. By using a classical trace-conditioning paradigm of the eyelid response, they found that trkB $B^{\mathrm{PLC} /+}$ animals learn less well than trkB ${ }^{\mathrm{SHC} /+}$ or control animals. When the hippocampal CA3 region was stimulated during the trace-conditioning paradigm, recording the field excitatory postsynaptic potential (fEPSP) in the CA1 region revealed an increase in synaptic strength in trkB ${ }^{\mathrm{SHC} /+}$ and control animals, but not in trkB $B^{\mathrm{PLC} /+}$ animals. Furthermore, the linear relationship between the acquisition of the eyelid response and potentiation at the $\mathrm{CA} 3-\mathrm{CA} 1$ synapses in control and trkB ${ }^{\mathrm{SHC} /+}$ animals was completely absent in trkB $B^{\mathrm{PLC} /+}$ animals, indicating that the PLC $\gamma$ docking site of TrkB is key to both processes. Further experiments investigating short-term and long-term effects after stimulation provided evidence that the $\operatorname{TrkB}$ PLC $\gamma$ docking site is crucial for LTP in vivo.

This paper shows for the first time that the same molecular mechanism forms the basis for learning a task and for changes in synaptic plasticity seen during LTP in awake animals. Future studies could apply this combination of methods to other mouse models and decipher other signalling pathways that are involved in learning and LTP.

Claudia Wiedemann

ORIGINAL RESEARCH PAPER Gruart, A. et al. Mutation at the TrkB PLCg-docking site affects hippocampal LTP and associative learning in conscious mice. Learn. Mem. 14, 54-62 (2007)

DOI: 